Check for updates

The BMJ

Cite this as: $B M J 2021 ; 375: n 3064$ http://dx.doi.org/10.1136/bmi.n3064 Published: 10 December 2021

\section{Covid-19: Monoclonal antibodies authorised in US as alternative to vaccines for certain groups}

\section{Zosia Kmietowicz}

The US drug regulator has granted emergency use authorisation to a combination of two monoclonal antibodies to prevent covid-19 in adults and children over 12 who are immunocompromised and may not produce an adequate response to covid vaccines and for those in whom vaccination is not recommended.

Evusheld, which is produced by AstraZeneca and given as two intramuscular injections, contains the long acting monoclonal antibodies tixagevimab and cilgavimab. Studies suggest it could prevent infection for up to six months, but it should not be used instead of vaccination where these can be given, said Patrizia Cavazzon, director of the Center for Drug Evaluation and Research at the Food and Drug Administration (FDA). "Vaccines have proven to be the best defence available against covid-19," she said.

Tixagevimab and cilgavimab target different sites of the spike protein of SARS-CoV-2, designed to block the virus' attachment and entry into human cells.

Evidence to support Evusheld's emergency use authorisation comes from Provent, a randomised, double blind, placebo controlled clinical trial of over 5000 unvaccinated adults with a chronic medical condition or an increased risk of covid infection. ${ }^{1}$ Over 183 days Evusheld reduced the rate of symptomatic infection compared with placebo from $1 \%$ to $0.2 \%$, a relative risk reduction of almost $80 \%$.

Evusheld's possible side effects include hypersensitivity reactions (including anaphylaxis), bleeding at the injection site, headache, fatigue, and cough, according to the emergency use authorisation.

The FDA said that serious cardiac adverse events were infrequent in Provent, although more trial participants who received Evusheld had serious cardiac adverse events, such as myocardial infarction and heart failure, compared with those on placebo. All these participants had risk factors for cardiac disease or a history of cardiovascular disease before taking part in the trial and it is not clear if Evusheld caused these cardiac adverse events, said the FDA.

Mene Pangalos, executive vice president of biopharmaceuticals research and development at AstraZeneca, said the company was working to establish Evusheld's efficacy against the omicron variant, but that it worked to neutralise all previous SARS-CoV-2 variants.

It is thought that about $2 \%$ of the global population is at increased risk of an inadequate response to a covid-19 vaccine and could benefit from pre-exposure prophylaxis, including seven million Americans.

AstraZeneca has agreed to supply the US government with 700 ooo doses of Evusheld. The US government has indicated that it plans to distribute these doses to states and territories at no cost and on a pro rata basis.

The Medicines and Healthcare Products Regulatory Agency (MHRA) has approved two monoclonal antibodies-the combination Ronapreve (casirivimab and imdevimab) ${ }^{1}$ and Xevudy (sotrovimab) ${ }^{2}$-for use in the UK as treatments, but not for use as prophylaxis.

Penny Ward, an independent pharmaceutical physician and visiting professor in pharmaceutical medicine at King's College London, said, “Given that most immunosuppressed people may not respond well to vaccination and so remain at risk of developing covid if exposed despite vaccination this additional level of protection would considerably reduce their risk and enable them to return to a more normal life. A critical feature of this product is its long lasting effect, one shot being effective for at least six months based on currently available data and potentially for a longer period as the trial is ongoing."

She said that the MHRA is currently reviewing Evusheld. "Given the benefit that such a product can provide, an early decision is much to be desired, particularly if retained antiviral activity against the omnicron strain is confirmed.”

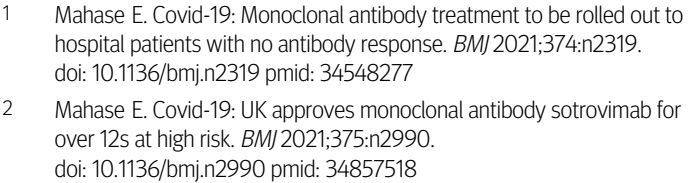

This article is made freely available for use in accordance with BMJ's website terms and conditions for the duration of the covid-19 pandemic or until otherwise determined by BMJ. You may use, download and print the article for any lawful, non-commercial purpose (including text and data mining) provided that all copyright notices and trade marks are retained. 